\title{
Evaluation of Petroleum Spray Oil Against Turnip Aphid, Lipaphis erysimi (Kaltenbach) Infesting Oilseed Brassica
}

\author{
Sarwan Kumar \\ Dept. of Plant Breeding and Genetics, Punjab Agricultural University, Ludhiana (141 004), India
}

\section{Article History}

Manuscript No. AR1358b

Received in $4^{\text {th }}$ April, 2015

Received in revised form $29^{\text {th }}$ September, 2015

Accepted in final form $7^{\text {th }}$ October, 2015

\section{Correspondence to}

${ }^{*} E$-mail: sarwanent@pau.edu

\section{Keywords}

Coccinella septempunctata, eco-friendly, mustard aphid, rapeseed-mustard

\begin{abstract}
Petroleum spray oil was evaluated against turnip aphid, Lipaphis erysimi (Kaltenbach) on Brassica juncea cv. PBR 210 at Punjab Agricultural University, Ludhiana during 2009-10 and 2010-11 crop seasons. Different treatments of petroleum spray oil included: 0.50, 0.75, 1.00, 1.25, 1.50 and 1.75\% along with dimethoate 30 EC @ 1 lt $\mathrm{ha}^{-1}$ and untreated control. All the treatments resulted in significant reduction in the aphid population after 3 days of spray except petroleum spray oil $(0.5 \%)$ and remained effective for up-to 10 days. Though, the chemical insecticide dimethoate was the most effective, petroleum spray oil @ 1.75\% was statistically at par with it with 82.2-86.9\% reduction in the aphid population and consistently remained effective for up to 10 days after application. Further, petroleum spray oil at either of the concentrations did not result in any toxic effect on Coccinella septempunctata and honey bees. The seed yield in the case of petroleum spray oil at all the concentrations, except at $0.5 \%$, was statistically at par with the chemical insecticide dimethoate (1 $\left.1 \mathrm{lt} \mathrm{ha}^{-1}\right)$ in 2009-10, however, in 2010-11, the yield differences were non-significant due to low aphid population. Keeping in view its efficacy, petroleum spray oil has the potential to be used as an alternative to chemical insecticides for the management of turnip aphid.
\end{abstract}

\section{Introduction}

The members of the family Brassicaceae are cultivated throughout world for food, oil and feed purposes. Among these, oilseed brassicas are important source of oil and protein and India is one of the largest producers of rapeseed-mustard including China and Canada (FAOSTAT, 2009). Brassica juncea is the major winter season oilseed crop cultivated in India, while other species like B. napus and B. rapa are grown to a limited extent. Among the biotic stresses that confront these crops, the damage caused by mustard/turnip aphid, Lipaphis erysimi (Kaltenbach) (Hemiptera: Aphididae) is the major constraint in realization of full yield potential of the crop (Kumar et al., 2011; Atri et al., 2012). The damage caused by this key pest of mustard ranges from 35.0 to $91.0 \%$ in different agro-climatic conditions of the country (Singh and Sachan, 1994) and results in complete crop failure in the event of lack of control measures.

Because of the increasing public and scientific concerns about the known adverse effects of toxic insecticidal chemicals and risks associated with their use, the need of alternative methods of pest management is always sought which highlights the importance of this study. Among the alternatives of insect pest management, petroleum spray oils are considered as potential control agents against many insects. They are currently regarded as more environmental friendly than synthetic pesticides and are finding place in Integrated Pest Management (IPM) programs (Beattie and Smith, 1990), and degrade relatively quickly in the environment (Davidson et al., 1991; Beattie et al., 1995b) and have never been associated with resistance or outbreaks of secondary pests (Beattie, 1989, 1990). Keeping these in view, the present study was carried out with the objective to evaluate petroleum spray oil against mustard aphid infesting Indian mustard, Brassica juncea.

\section{Materials and Methods}

\subsection{Study site}

The study was carried out at Oilseeds Research Farm, Department of Plant Breeding and Genetics, Punjab Agricultural University, Ludhiana, $\left(30.9^{\circ} \mathrm{N}\right.$ and $75.85^{\circ} \mathrm{E}, 244$ $\mathrm{m}$ above $\mathrm{msl}$ ), India during 2009-10 and 2010-11 crop seasons.

\subsection{Treatments and plant material}

Brassica juncea cv. PBR 210 was sown in plots of size $4.2 \times 3.0 \mathrm{~m}^{2}$ with row to row and plant to plant spacing of 
30 and $15 \mathrm{~cm}$, respectively. The experiment was laid out in Randomized Complete Block Design with eight treatments and three replications. All the recommended package of practices was followed for raising a good crop except for spray of insecticides (PAU, 2009).

Different treatments were applied when the mustard aphid population reached economic threshold level of 50-60 aphids/ plant (PAU, 2009). During 2009-10, two sprays were applied while in 2010-11 only one spray could be applied owing to low aphid population. The treatments included mustard spray oil from Hindustan Petroleum @ 0.5, 0.75, 1.00, 1.25, 1.50 and $1.75 \%$ along with dimethoate $30 \mathrm{EC} @ 11 \mathrm{lt} \mathrm{ha}^{-1}$ and untreated control.

Under laboratory conditions, toxicity of different treatments to coccinellid beetles was evaluated. For this, treated leaves were collected from the field which harboured aphid colonies on their lower surface and placed in Petri plates slightly overlapping one over the other to facilitate contact to the treated surface. Five adults of Coccinella septempuncata were released in each Petri plate. The experiment was laid out in Completely Randomized Design with three replications per treatment. The leaves along with aphids were changed every alternate day.

\subsection{Data collection and statistical analysis}

Data on the aphid population/plant were recorded from top $10 \mathrm{~cm}$ central twig of plant before, 1, 3, 5, 7, 10 and 14 days after treatment from 10 plants selected at random from each plot. In 2010-11, the population of mustard aphid was very low, hence, data on the aphid population were recorded from tagged infested plants in the field. In addition, data on the number of bee visits plant ${ }^{-1}$ minute $^{-1}$ were also recorded. Data on number of grubs and adults of coccinellids plant ${ }^{-1}$ were also recorded from 10 plants selected at random from each plot. Yield data were recorded at harvest of the crop. Data of the two years were not pooled due to non-significant differences in yield among different treatments in the year 2010-11. In the laboratory experiment, mortality data of $C$. septempunctata were recorded every 24 hours for five days after their release. The field data on mustard aphid population, honey bees activity, coccinellid population and yield data were subjected to analysis of variance following randomized complete block design while the laboratory data on mortality of coccinellid beetles were subjected to analysis of variance following completely randomized design using the statistical software OPSTAT (OPSTAT, 2009). Means were separated by least significant difference (LSD) at 5\% level of significance.

\section{Results and Discussion}

All the treatments resulted in reduction in aphid population and provided protection from mustard aphid for 10 days.
After one day of treatment, there was significant decline in aphid population over control in all the treatments except HP mustard spray oil @ 0.50\% (Table 1). In the year 2009-10, the minimum population of 12.3 aphids plant ${ }^{-1}$ was recorded in dimethoate30 EC @ 1 1t ha ${ }^{-1}$ followed by HP mustard spray oil @ 1.75 and 1.50\% (14.7 aphids plant ${ }^{-1}$ each). After 3 days of treatment, aphid population in all the treatments was significantly lower than that in the control. The minimum aphid population was observed in the chemical insecticide dimethoate@ 1 lt ha $^{-1}$ (6.6 aphids plant $\left.{ }^{-1}\right)$. The aphid population in the HP mustard spray oil treatment $1.75 \%$ and $1.50 \%$ (12.5 and 14.3 aphids plant ${ }^{-1}$, respectively) was at par with the chemical insecticide dimethoate @ 1 1t ha ${ }^{-1}$ (6.6 aphids/ plant) and significantly lower than the remaining treatments of HP mustard spray oil. Almost similar trend was observed after 5, 7 and 10 days of treatment. The spray oil at 1.50 and $1.75 \%$ remained as effective as chemical insecticide for up-to 10 days after treatment. The second spray was done after 15 days of first spray. Almost similar trend as observed after first spray was observed after second spray with respect to efficacy of different treatments after 1 day of second spray. However, due to light thunderstorms, there was a sudden decline in the aphid population which did not develop further.

The trend in the efficacy of different treatments in 2010-11 crop was almost similar to that observed in 2009-10. After 1 day of treatment, the minimum aphid population of 10.2 aphids plant $^{-1}$ was recorded in the case of dimethoate (Table 1). It was followed by petroleum oil @ 1.75 and 1.50\% (13.5 and 15.0 aphids plant ${ }^{-1}$, respectively) which were at par with chemical insecticide. After 3 days of treatment, aphid population in all the treatments was significantly lower than control. The minimum aphid population was observed in dimethoate (4.8 aphids plant $\left.{ }^{-1}\right)$. It was followed by petroleum spray oil @ 1.75 and $1.50 \%$ (11.8 and 12.3 aphids plant ${ }^{-1}$, respectively). Almost similar trend was observed after 5, 7 and 10 days of treatment. The spray oil at $1.75 \%$ remained as effective as chemical insecticide in reducing aphid population for up-to 10 days after application. Since the aphid population started declining after 10 days of first spray, second spray could not be applied. As the population of mustard aphid was very low and only a few plants in a plot were found infested, therefore, yield differences among treatments were non-significant.

Data on the toxicity of different treatments to honeybees are presented in (Table 2). Although slight reduction in bees' activity was recorded in all the treatments following spray including the chemical insecticide dimethoate during both the years, but statistically it was non-significant when compared with untreated control. Almost similar trend was observed with respect to toxicity of different treatments to natural enemies during both the years i.e. the treatments were statistically 
Table 1: Efficacy of petroleum spray oil against mustard aphid under field conditions during 2009-10 and 2010-11

Sr. Treatment

Aphid population plant $^{-1}($ Mean \pm SE)

Yield

no.

\begin{tabular}{|c|c|c|c|c|c|c|c|c|c|c|c|c|c|c|c|c|}
\hline \multicolumn{16}{|c|}{ Aphid population plant $^{-1}(\mathrm{Mean} \pm \mathrm{SE})$} & \multirow{2}{*}{$\begin{array}{l}\text { Yield } \\
\text { (kg ha- }^{-1}\end{array}$} \\
\hline \multicolumn{9}{|c|}{$2009-10$} & \multicolumn{7}{|c|}{ 2010-11 } & \\
\hline " & 充 & 㐫 & $\begin{array}{l}\text { 峞 } \\
\text { in }\end{array}$ & 离 & 䎛 & 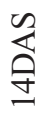 & 岕 & $\frac{\infty}{\infty}$ & $\tilde{m}$ & 斑 & 禹 & 岕 & $\stackrel{\infty}{\stackrel{\infty}{0}}$ & 先 & 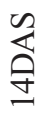 & 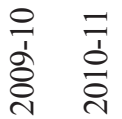 \\
\hline
\end{tabular}

$\left(\mathrm{kg} \mathrm{ha}^{-1}\right)$

1. Petroleum spray oil (a) $0.50 \%$
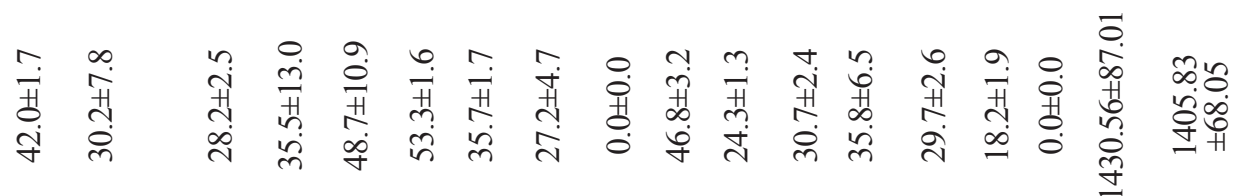

2. Petroleum spray oil (a) $0.75 \%$

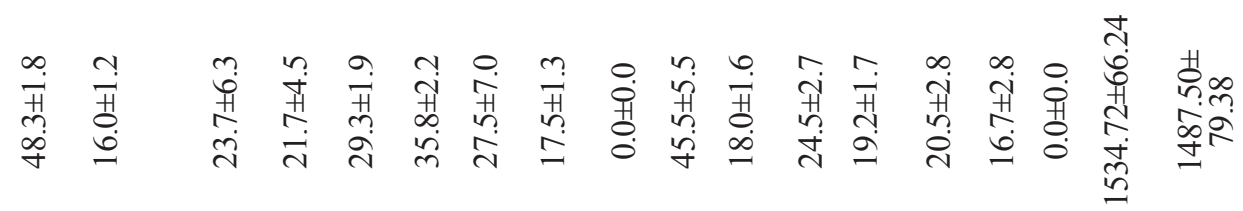

3. Petroleum spray oil (a) $1.00 \%$

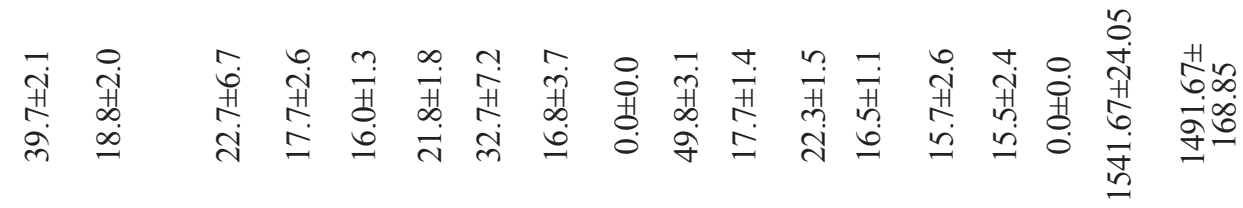

4. Petroleum spray oil (a) $1.25 \%$

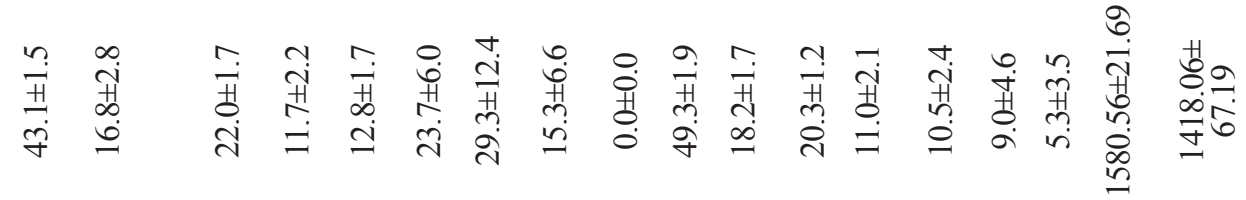

5. Petroleum spray oil (a) $1.50 \%$

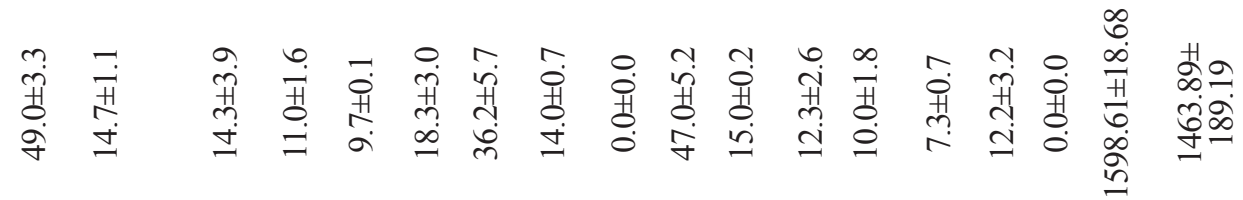

6. Petroleum spray oil (a) $1.75 \%$

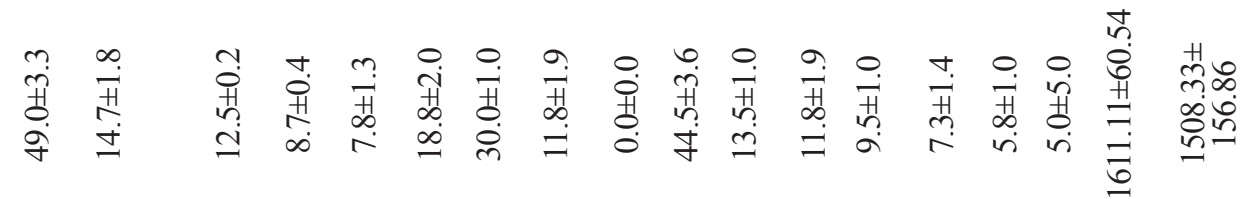

7. Dimethoate $30 \mathrm{EC}$ (a) 1 lt ha-

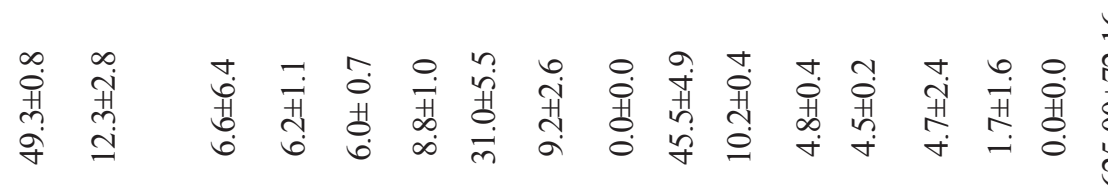

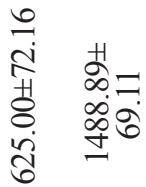

8. Control

$$
\mathrm{CD}(p=0.05)
$$

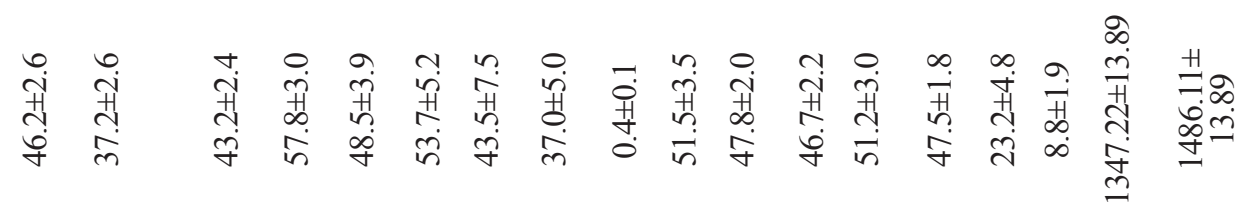

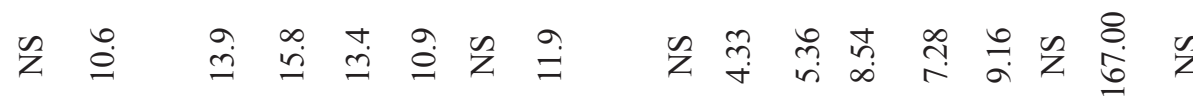

"BS: Before spray; $†$ DAS: Days after spray 
Table 2: Toxicity of petroleum spray oil to honey bees under field conditions during 2009-10 and 2010-11

\begin{tabular}{|c|c|c|c|c|c|c|c|c|c|c|c|c|c|c|c|}
\hline \multirow{4}{*}{$\begin{array}{l}\text { Sr. } \\
\text { no. }\end{array}$} & \multirow[t]{4}{*}{ Treatment } & \multicolumn{14}{|c|}{ Number of bee visits plot ${ }^{-1}$ minute $^{-1}$} \\
\hline & & \multicolumn{7}{|c|}{$2009-10$} & \multicolumn{7}{|c|}{$2010-11$} \\
\hline & & $\mathrm{BS}^{*}$ & 1 & 3 & 5 & 7 & 10 & 14 & BS & 1 & 3 & 5 & 7 & 10 & 14 \\
\hline & & & $\mathrm{DAS}^{\dagger}$ & DAS & DAS & DAS & DAS & DAS & & DAS & DAS & DAS & DAS & DAS & DAS \\
\hline 1. & $\begin{array}{l}\text { Petroleum spray oil } \\
\text { @ } 0.50 \%\end{array}$ & $\begin{array}{l}\dddot{m} \\
\stackrel{+}{+} \\
\text { ì }\end{array}$ & $\begin{array}{l}m \\
\stackrel{n}{0} \\
\text { ñ } \\
\text { in }\end{array}$ & $\begin{array}{l}\text { m. } \\
\text { H } \\
\text { f. }\end{array}$ & $\begin{array}{l}\stackrel{m}{o} \\
\stackrel{+}{r} \\
\sim\end{array}$ & $\begin{array}{l}m \\
? \\
\dot{H} \\
m \\
m\end{array}$ & $\begin{array}{l}0 \\
0 \\
0 \\
0 \\
0\end{array}$ & $\begin{array}{l}m \\
0 \\
0 \\
m \\
0\end{array}$ & $\begin{array}{l}n \\
0 \\
0 \\
\dot{0} \\
\dot{m}\end{array}$ & $\begin{array}{l}\text { n. } \\
0 \\
+1 \\
\text { I. }\end{array}$ & $\begin{array}{l}ت \\
\dot{H} \\
\dot{0} \\
\text { ن. }\end{array}$ & $\begin{array}{l}\infty \\
\stackrel{0}{0} \\
\dddot{H} \\
\sim \\
\sim\end{array}$ & $\begin{array}{l}0 \\
0 \\
0 \\
\dddot{H} \\
-\end{array}$ & $\begin{array}{l}0 \\
\stackrel{0}{0} \\
\stackrel{+}{1} \\
\stackrel{n}{n}\end{array}$ & $\begin{array}{l}n \\
0 \\
0 \\
0\end{array}$ \\
\hline 2. & $\begin{array}{l}\text { Petroleum spray oil } \\
\text { (a) } 0.75 \%\end{array}$ & $\begin{array}{l}\stackrel{0}{0} \\
\stackrel{+}{1} \\
\stackrel{i}{i}\end{array}$ & 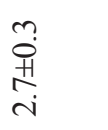 & $\begin{array}{l}n \\
0 \\
0 \\
0\end{array}$ & $\begin{array}{l}\dddot{m} \\
\stackrel{+}{+} \\
\text { I. }\end{array}$ & $\begin{array}{c}m \\
\stackrel{n}{+} \\
\stackrel{i}{i}\end{array}$ & $\begin{array}{l}m \\
\stackrel{n}{1} \\
\dddot{m}\end{array}$ & $\begin{array}{l}\stackrel{0}{0} \\
\stackrel{+}{+} \\
\stackrel{0}{0}\end{array}$ & $\begin{array}{l}\exists \\
\dot{H} \\
\dot{m}\end{array}$ & $\frac{\dot{H}}{\frac{\dot{H}}{i}}$ & $\begin{array}{l}n \\
0 \\
0 \\
0 \\
i\end{array}$ & $\frac{m}{\stackrel{+}{i}}$ & $\underset{m}{\text { N̦ }}$ & $\begin{array}{l}\stackrel{0}{0} \\
\stackrel{0}{H} \\
\dot{0}\end{array}$ & $\begin{array}{l}0 \\
\stackrel{0}{0} \\
0 \\
0\end{array}$ \\
\hline 3. & $\begin{array}{l}\text { Petroleum spray oil } \\
\text { (a) } 1.00 \%\end{array}$ & $\begin{array}{l}0 \\
\stackrel{0}{+} \\
\text { ñ } \\
i\end{array}$ & $\begin{array}{l}m \\
\stackrel{n}{0} \\
\text { ñ }\end{array}$ & $\begin{array}{l}n \\
0 \\
0 \\
0\end{array}$ & $\begin{array}{l}m \\
\stackrel{n}{0} \\
m \\
n\end{array}$ & $\begin{array}{l}n \\
0 \\
0 \\
0 \\
\text { in }\end{array}$ & $\begin{array}{l}\dddot{m} \\
\stackrel{+}{1} \\
0\end{array}$ & $\begin{array}{l}\stackrel{0}{0} \\
\stackrel{+}{0} \\
\stackrel{0}{0}\end{array}$ & $\begin{array}{l}m \\
\stackrel{+}{+} \\
\stackrel{+}{r}\end{array}$ & 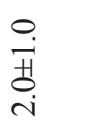 & 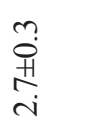 & $\begin{array}{l}n \\
0 \\
0 \\
0\end{array}$ & $\begin{array}{c}\stackrel{m}{0} \\
\stackrel{+}{i}\end{array}$ & $\begin{array}{l}0 \\
0 \\
0 \\
0\end{array}$ & 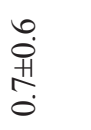 \\
\hline 4. & $\begin{array}{l}\text { Petroleum spray oil } \\
\text { (a) } 1.25 \%\end{array}$ & $\begin{array}{l}n \\
0 \\
0 \\
0 \\
\dot{y}\end{array}$ & 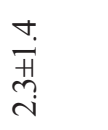 & 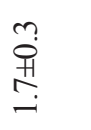 & $\begin{array}{l}0 \\
0 \\
\dot{0} \\
0 \\
\text { ن. }\end{array}$ & $\begin{array}{l}n \\
0 \\
0 \\
0 \\
i\end{array}$ & $\begin{array}{l}\stackrel{0}{0} \\
\stackrel{+}{+} \\
\stackrel{0}{0}\end{array}$ & 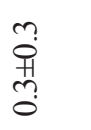 & $\begin{array}{l}\infty \\
\stackrel{0}{+} \\
\stackrel{+}{i}\end{array}$ & $\begin{array}{l}\stackrel{0}{\dot{d}} \\
\stackrel{+}{0} \\
\stackrel{i}{ }\end{array}$ & \begin{tabular}{l}
$n$ \\
$\stackrel{0}{0}$ \\
\multirow{1}{0}{} \\
i
\end{tabular} & $\begin{array}{l}m \\
\stackrel{1}{+} \\
\text { ñ }\end{array}$ & 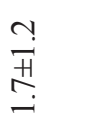 & 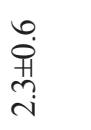 & $\begin{array}{l}0 \\
\stackrel{0}{0} \\
\stackrel{+}{0}\end{array}$ \\
\hline 5. & $\begin{array}{l}\text { Petroleum spray oil } \\
\text { (a) } 1.50 \%\end{array}$ & $\begin{array}{l}n \\
0 \\
0 \\
0 \\
i\end{array}$ & $\begin{array}{l}0 \\
0 \\
\stackrel{+}{1} \\
\stackrel{n}{n}\end{array}$ & $\begin{array}{l}n \\
0 \\
0 \\
0 \\
\text { ¿̇ }\end{array}$ & $\begin{array}{l}\infty \\
0 \\
+ \\
\stackrel{+}{I} \\
-\end{array}$ & $\begin{array}{l}n \\
0 \\
0 \\
0\end{array}$ & $\begin{array}{l}0 \\
0 \\
0\end{array}$ & $\begin{array}{l}\stackrel{0}{0} \\
\stackrel{0}{1} \\
0 \\
0\end{array}$ & $\frac{\text { N }}{\text { H }}$ & 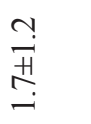 & $\begin{array}{l}\stackrel{0}{\dot{d}} \\
\text { ț } \\
\stackrel{i}{ }\end{array}$ & $\begin{array}{l}n \\
0 \\
0 \\
0 \\
\dot{+}\end{array}$ & $\begin{array}{l}m \\
\stackrel{1}{H} \\
\text { ñ }\end{array}$ & $\begin{array}{l}0 \\
\stackrel{0}{0} \\
\text { Ḣ } \\
\text { i }\end{array}$ & $\begin{array}{l}n \\
0 \\
+1 \\
0\end{array}$ \\
\hline 6. & $\begin{array}{l}\text { Petroleum spray oil } \\
\text { (a) } 1.75 \%\end{array}$ & $\begin{array}{l}\infty \\
\stackrel{0}{+} \\
\stackrel{+}{亡} \\
-\end{array}$ & $\begin{array}{l}n \\
0 \\
0 \\
0 \\
ن\end{array}$ & $\begin{array}{l}0 \\
\stackrel{0}{+1} \\
\text { ñ }\end{array}$ & $\begin{array}{l}m \\
\stackrel{n}{+} \\
\tilde{n}\end{array}$ & $\begin{array}{l}\infty \\
\stackrel{0}{+} \\
\text { m. } \\
-\end{array}$ & $\begin{array}{l}n \\
0 \\
0 \\
0\end{array}$ & 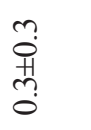 & $\begin{array}{l}\stackrel{0}{0} \\
\stackrel{+}{1} \\
\stackrel{i}{1}\end{array}$ & 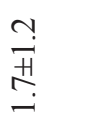 & $\frac{\text { Nִ }}{\stackrel{H}{I}}$ & $\begin{array}{l}\infty \\
\stackrel{0}{+} \\
\text { ஸे } \\
ن\end{array}$ & $\begin{array}{l}\infty \\
\stackrel{\infty}{+} \\
\stackrel{+}{-}\end{array}$ & 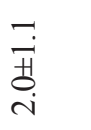 & $\begin{array}{l}n \\
0 \\
0 \\
0\end{array}$ \\
\hline 7. & $\begin{array}{l}\text { Dimethoate } 30 \mathrm{EC} \\
\text { (a) } 1 \mathrm{lt} \mathrm{ha}^{-1}\end{array}$ & 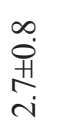 & $\begin{array}{l}n \\
\stackrel{0}{0} \\
0 \\
0\end{array}$ & $\begin{array}{l}n \\
\dddot{m} \\
\text { ṇ }\end{array}$ & $\begin{array}{l}n \\
\text { no } \\
0 \\
\dot{n}\end{array}$ & $\begin{array}{l}m \\
\stackrel{1}{+} \\
\stackrel{1}{I}\end{array}$ & 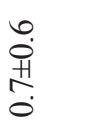 & $\begin{array}{l}n \\
\stackrel{0}{0} \\
0 \\
0\end{array}$ & $\begin{array}{l}\infty \\
0 \\
+ \\
m \\
m\end{array}$ & $\begin{array}{l}0 \\
\stackrel{0}{\oplus} \\
\stackrel{m}{-}\end{array}$ & $\begin{array}{l}n \\
\stackrel{0}{0} \\
0\end{array}$ & $\frac{\text { N̦ }}{\text { On }}$ & $\begin{array}{l}\vec{H} \\
\stackrel{H}{+} \\
\stackrel{\sim}{i}\end{array}$ & $\begin{array}{l}\infty \\
\stackrel{\infty}{\dagger} \\
\stackrel{1}{I}\end{array}$ & 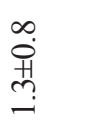 \\
\hline 8. & Control & $\begin{array}{l}\infty \\
\stackrel{\infty}{0} \\
\stackrel{1}{n} \\
\stackrel{1}{n}\end{array}$ & $\begin{array}{l}\stackrel{0}{0} \\
\stackrel{1}{+} \\
\stackrel{+}{+}\end{array}$ & $\begin{array}{l}\infty \\
\stackrel{1}{0} \\
\stackrel{n}{\sim}\end{array}$ & $\underset{\stackrel{\infty}{+}}{\stackrel{\infty}{\sim}}$ & 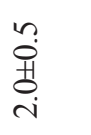 & $\begin{array}{l}\text { m? } \\
\text { H1 } \\
\text { m }\end{array}$ & $\begin{array}{l}0 \\
\stackrel{0}{0} \\
\stackrel{0}{0} \\
0\end{array}$ & $\begin{array}{l}n \\
0 \\
00 \\
\end{array}$ & $\begin{array}{l}\stackrel{0}{0} \\
\stackrel{1}{1} \\
m \\
+\end{array}$ & $\begin{array}{l}n \\
0 \\
0 \\
0 \\
i\end{array}$ & $\begin{array}{l}n \\
0 \\
0 \\
0 \\
\text { ñ }\end{array}$ & $\begin{array}{l}\infty \\
\stackrel{\infty}{\oplus} \\
\stackrel{1}{n} \\
\stackrel{1}{0}\end{array}$ & 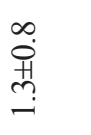 & $\begin{array}{l}\text { m? } \\
\text { †1 } \\
\text { m? }\end{array}$ \\
\hline & $\mathrm{CD}(p=0.05)$ & Z & Z & Z & 反 & Z & $\tilde{z}$ & Z & Z & Z & $\tilde{z}$ & $\tilde{z}$ & Z & Z & Z \\
\hline
\end{tabular}

*BS: Before spray; $†$ DAS: Days after spray

non-significant (Table 3). Although, larvae of Chrysoperla carnea and syrphid fly were observed in the later part of the season, but the population was in traces and too low to draw valid conclusions. Therefore, the data for coccinellid beetles only are presented. There were non significant differences in the population of coccinellid beetles in different treatments (Table 3).

Under the laboratory conditions, HP mustard spray oil did not result in significant mortality of coccinellid beetles compared to control for up-to 120 hours after treatment (Table 4). However, more than 60 and $80 \%$ mortality was observed in dimethoate in 2009-10 and 2010-11, respectively, which was significantly higher than that in the control.

In the year 2009-10, the seed yield in all the treatments was significantly higher than that in the control $\left(1347.2 \mathrm{k} \mathrm{ha}^{-1}\right)$ except in petroleum spray oil @ $0.5 \%\left(1430.56 \mathrm{k} \mathrm{ha}^{-1}\right)($ Table
1). The maximum seed yield of $1625.00 \mathrm{k} \mathrm{ha}^{-1}$ was recorded in the case of dimethoate. It was followed by petroleum spray oil @ 1.75\% (1611.11 k ha-1), 1.50\% (1598.61 k ha $\left.{ }^{-1}\right), 1.25 \%$ $\left(1580.56 \mathrm{k} \mathrm{ha}^{-1}\right), 1.00 \%\left(1541.67 \mathrm{k} \mathrm{ha}^{-1}\right)$ and $0.75 \%(1534.72$ $\mathrm{k} \mathrm{ha}^{-1}$ ) which were at par with each other. However, in the year 2010-11, the population of mustard aphid was very low and hence, the yield differences among the treatments were non-significant. Thus, it can be concluded from the study that petroleum spray oil @ 1.75\% resulted in significant reduction in aphid population for up-to 10 days of treatment in both the years and was at par with chemical insecticide dimethoate @ $1 \mathrm{ml} \mathrm{ha}^{-1}$. Further, it did not result in any significant adverse effect on both coccinellid predators as well as honey bees.

Petroleum spray oils are very effective in controlling a range of insect-pests such as aphids, scale insects, mites etc. (Davidson et al., 1991; Lawson and Weires, 1991). In the present study, 
Table 3: Toxicity of petroleum spray oil to coccinellids under field conditions during 2009-10 and 2010-11

Sr. Treatment Number of Coccinellid beetles plant ${ }^{-1}$

no.

\begin{tabular}{cccccccccccccccccc}
\hline \multicolumn{10}{c}{$2009-10$} & & \multicolumn{8}{c}{$2010-11$} \\
\hline BS $^{*}$ & 1 & 3 & 5 & 7 & 10 & 14 & 1 & 3 & & BS & 1 & 3 & 5 & 7 & 10 & 14 \\
& DAS $\dagger$ & DAS & DAS & DAS & DAS & DAS & DAS & DAS & & DAS & DAS & DAS & DAS & DAS & DAS \\
\hline
\end{tabular}

1. Petroleum spray oil (a) $0.50 \%$

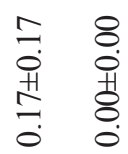

\begin{tabular}{ll}
0 & \multicolumn{1}{c}{} \\
0 & 0 \\
0 & 0 \\
0 & +1 \\
0 & 0 \\
0 & 0
\end{tabular}

\begin{tabular}{ll}
0 & \multicolumn{2}{c}{} \\
0 & 0 \\
0 & 0 \\
0 & 0 \\
0 & 0 \\
0 & 0
\end{tabular}

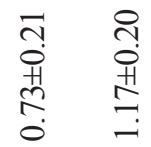

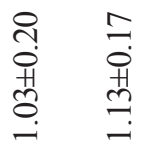

$\begin{array}{ll}8 & 8 \\ 0 & 0 \\ 0 & 0 \\ \text { 웅 } \\ 0 & 0 \\ 0 & 0\end{array}$

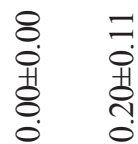

?̊.

$\begin{array}{ll}= & 0 \\ 0 & 0 \\ 0 & +1 \\ \text { H1 } & 0 \\ 0 & 0\end{array}$

2. Petroleum $\begin{array}{ll}8 & 8 \\ 0 & 0 \\ 0 & \text { H1 } \\ 0 & 0 \\ 0 & 0\end{array}$

$\begin{array}{ll}m & 0 \\ 0 & 0 \\ 0 & 0 \\ 0 & 0 \\ 0 & 0 \\ 0 & 0\end{array}$

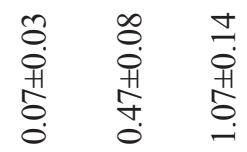

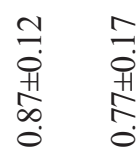

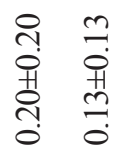

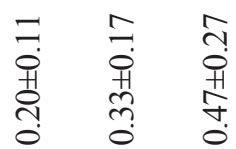

$\begin{array}{ll}\text { กิ } & 8 \\ 0 & 0 \\ \text { 11 } & 8 \\ 0 & 8 \\ 0 & 0\end{array}$

3. Petroleum spray oil (a) $1.00 \%$

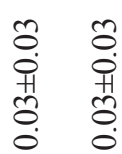

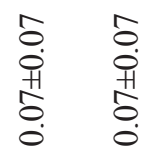

8
0
0
0
0

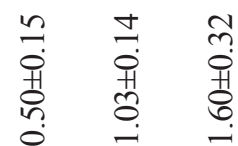

$\begin{array}{lll}n & 8 & 8 \\ 0 & 0 & 0 \\ 0 & 01 & 11 \\ 0 & 8 & 0 \\ 0 & 0 & 0\end{array}$

$\begin{array}{lll}5 & 8 & m \\ 0 & 0 & 0 \\ 01 & 01 & 1 \\ 0 & 0 & m \\ 0 & 0 & 0\end{array}$

$\begin{array}{ll}5 & 8 \\ 0 & 0 \\ 0 & 0 \\ \text { 11 } & 8 \\ 0 & 0 \\ 0 & 0\end{array}$

4. Petroleum spray oil (a) $1.25 \%$

$\begin{array}{ll}8 & 8 \\ 0 & 0 \\ 01 & 8 \\ 0 & 0 \\ 0 & 0\end{array}$

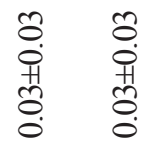

$\begin{array}{ll}\infty & = \\ 0 & 0 \\ 0 & 0 \\ 0 & 8 \\ 0 & 0\end{array}$

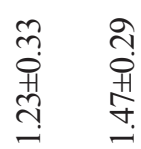

$\begin{array}{ll}8 & 5 \\ 0 & 0 \\ 0 & 0 \\ 0 & 0 \\ 0 & 0 \\ 0 & 0\end{array}$

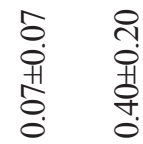

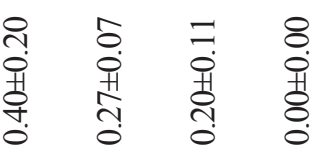

5. Petroleum spray oil (a) $1.50 \%$

$\begin{array}{ll}5 & 8 \\ 0 & 0 \\ 0 & 0 \\ 0 & 0 \\ 0 & 0\end{array}$

$\begin{array}{ll}8 & n \\ 0 & 0 \\ 01 & 01 \\ 8 & 0 \\ 0 & 0\end{array}$

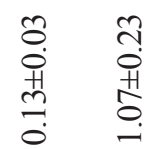

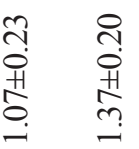

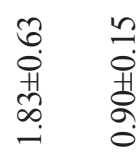

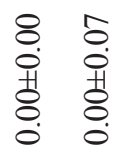

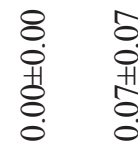

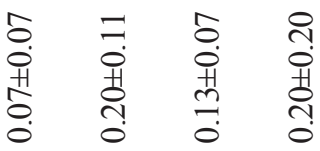

6. Petroleum spray oil (a)1.75\%

$\begin{array}{ll}\circ & 0 \\ 0 & 0 \\ 01 & +1 \\ 0 & 0 \\ 0 & 0\end{array}$

\begin{tabular}{|c|}
\hline \\
\hline $\begin{array}{l}0 \\
\text { क्ष } \\
\text { ô. }\end{array}$ \\
\hline
\end{tabular}

$\infty$
0
0


0

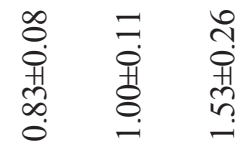

ํㅜㅇ

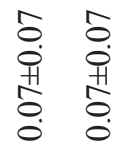

चे

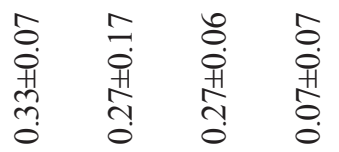

7. Dimetho-

ate $30 \mathrm{EC}$

$\begin{array}{ll}8 & 8 \\ 0 & 0 \\ 01 & 0 \\ 0 & 8 \\ 0 & 0\end{array}$

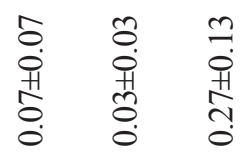

$\begin{array}{ll}\infty & = \\ 0 & 0 \\ 01 & 01 \\ 03 & \text { ஸे } \\ 0 & -1\end{array}$

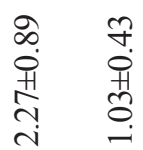

$\begin{array}{ll}8 & 8 \\ 0 & 0 \\ 0 & 0 \\ 1 & 8 \\ 0 & 0 \\ 0 & 0\end{array}$

\begin{tabular}{lllll}
8 & $m$ & \multicolumn{1}{c}{} & $m$ & 8 \\
0 & 0 & 0 & 0 & 0 \\
01 & +1 & +1 & 1 & 11 \\
8 & $m$ & 0 & $m$ & 0 \\
0 & 0 & 0 & 0 & 0
\end{tabular}

8. Control

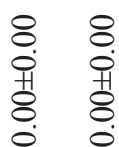

$\begin{array}{ll}0 & 0 \\ 0 & 0 \\ 01 & +1 \\ 0 & 0 \\ 0 & 0\end{array}$

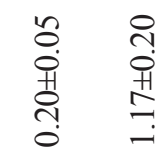

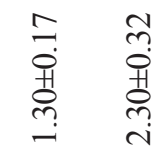

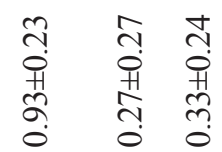

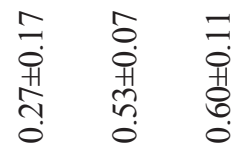

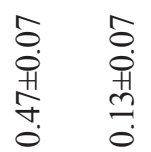

$\mathrm{CD}$ $(p=0.05)$

*BS: Before spray; $\uparrow$ DAS: Days after spray

the spray oil at $1.75 \%$ concentration consistently remained as effective as chemical insecticide for up-to 10 days after application in both the years. The major tenet of IPM is the reduced use of pesticides and the use of alternative pest management options least disruptive to the environment. It is a well known fact that oils can result in insect mortality by physically interfering with their respiration as well as by chemical action (Martin and Woodcock, 1983). Mineral oils are being used for insect pest management for over two centuries (Miller, 1983; Agnello, 2002) and continue to play an important role in insect pest management in fruit trees in the United States (Riehl, 1981), Australia (Furness and Maelzer, 1981), Israel (Neubauer, 1981), Japan (Ohkubo, 1981) and Canada (Anonymous, 1991). However, there is scanty information about their use on mustard crop and the present work attempts to fill this gap.

Phytotoxicity is a major limitation of petroleum oils as crop protectants. But in the present study, no phytotoxicity was observed even at the highest concentration of $1.75 \%$. Petroleum spray oil is a refined petroleum product that is distilled to 


\begin{tabular}{|c|c|c|c|c|c|c|c|c|c|c|c|}
\hline \multirow{3}{*}{$\begin{array}{l}\text { Sr. } \\
\text { no. }\end{array}$} & \multirow[t]{3}{*}{ Treatment } & \multicolumn{10}{|c|}{$\%$ Cumulative mortality (Hrs. after treatment) } \\
\hline & & \multicolumn{5}{|c|}{$2009-10$} & \multicolumn{5}{|c|}{$2010-11$} \\
\hline & & 24 & 48 & 72 & 96 & 120 & 24 & 48 & 72 & 96 & 120 \\
\hline 1. & Petroleum spray oil @ 0.50\% & 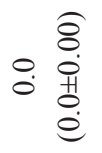 & 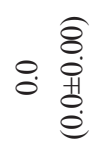 & 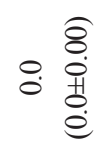 & 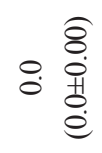 & 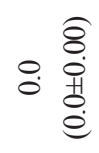 & 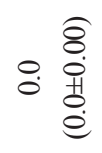 & 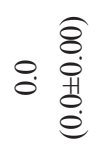 & 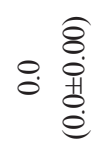 & 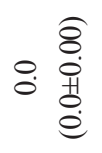 & $\begin{array}{ll} & \text { 8̊ } \\
\circ & \stackrel{0}{0} \\
0 & \dot{0} \\
& \dot{0}\end{array}$ \\
\hline 2. & Petroleum spray oil @ 0.75\% & 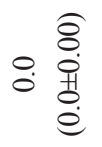 & 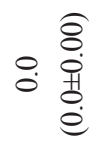 & 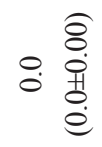 & 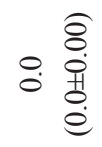 & 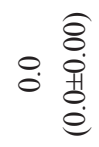 & 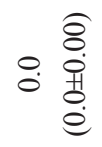 & 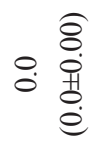 & 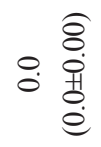 & 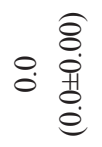 & 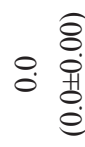 \\
\hline 3. & Petroleum spray oil @ 1.00\% & 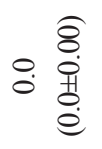 & 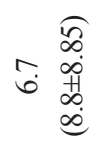 & 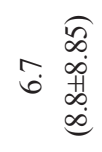 & 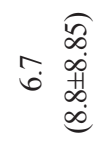 & 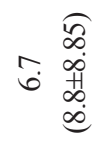 & 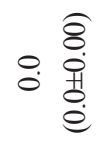 & $\begin{array}{ll} & \stackrel{8}{8} \\
\circ & \stackrel{0}{0} \\
0 & 1 \\
0 & \stackrel{0}{0}\end{array}$ & 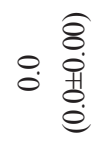 & 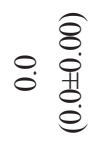 & 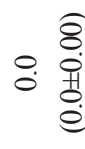 \\
\hline 4. & Petroleum spray oil @ 1.25\% & 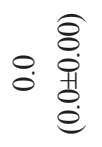 & 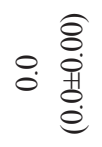 & 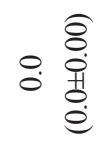 & 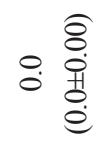 & 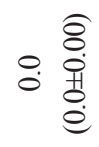 & 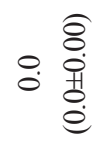 & 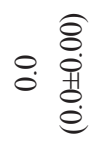 & 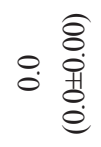 & 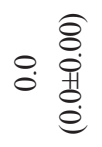 & 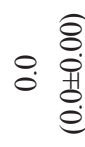 \\
\hline 5. & Petroleum spray oil @ 1.50\% & 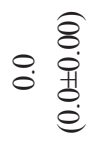 & 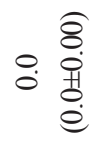 & 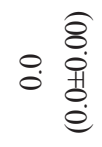 & 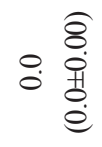 & 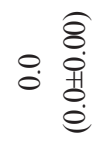 & 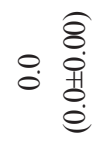 & 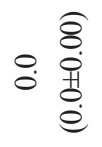 & 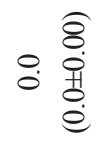 & 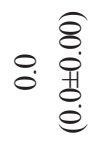 & 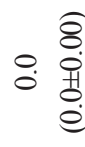 \\
\hline 6. & Petroleum spray oil @ 1.75\% & 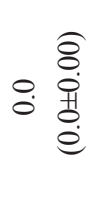 & 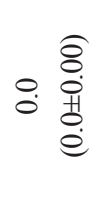 & 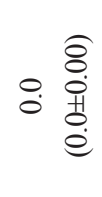 & 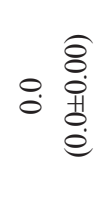 & $\begin{array}{l}0 \\
8 \\
0 \\
0 \\
0 \\
0 \\
0 \\
0 \\
0\end{array}$ & 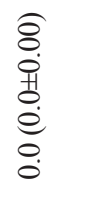 & 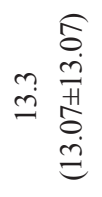 & 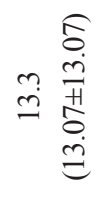 & 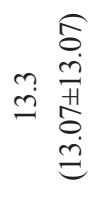 & 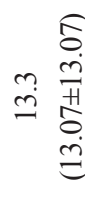 \\
\hline 7. & Dimethoate30 EC @ 1lt ha ${ }^{-1}$ & 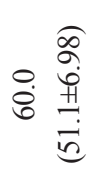 & 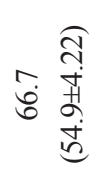 & 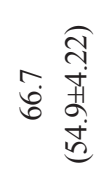 & 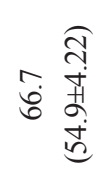 & 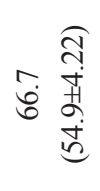 & 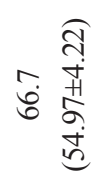 & 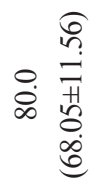 & 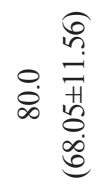 & 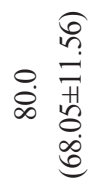 & 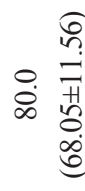 \\
\hline 8. & Control & $\begin{array}{l}\widehat{n} \\
\infty \\
0 \\
0 \\
0 \\
\infty \\
\infty \\
\infty\end{array}$ & 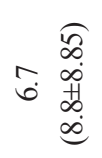 & $\begin{array}{r}\widehat{n} \\
\infty \\
0 \\
0 \\
0 \\
\infty \\
\infty \\
\infty\end{array}$ & 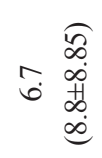 & 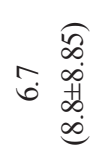 & 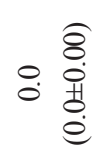 & 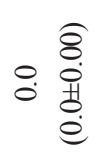 & 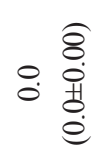 & 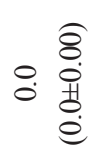 & 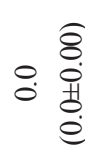 \\
\hline \multicolumn{2}{|c|}{$\mathrm{CD}(p=0.05)$} & $\stackrel{\sqrt{8}}{\stackrel{1}{\Xi}}$ & $\begin{array}{l}\overparen{I} \\
\stackrel{ \pm}{ \pm}\end{array}$ & $\begin{array}{l}\underset{I}{J} \\
\stackrel{\Xi}{\Xi}\end{array}$ & $\begin{array}{l}\underset{I}{J} \\
\underset{\Xi}{\Xi}\end{array}$ & $\begin{array}{l}\overparen{I} \\
\stackrel{ \pm}{ \pm}\end{array}$ & 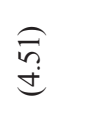 & $\begin{array}{l}\mathbb{8} \\
\infty \\
\stackrel{0}{=}\end{array}$ & $\begin{array}{l}\mathbb{8} \\
\stackrel{\infty}{=}\end{array}$ & $\begin{array}{l}\underset{8}{0} \\
\stackrel{0}{=}\end{array}$ & $\begin{array}{l}\overparen{8} \\
\stackrel{\infty}{=}\end{array}$ \\
\hline
\end{tabular}

Figures in parentheses are arc sin transformed values

remove impurities that can damage plants. Highly refined narrow range petroleum spray oils in particular are IPMcompatible pest control compounds (Beattie, 1991; Nicetic et al., 2001) that are effective against a wide range of pests (Davidson et al., 1991; Lawson and Weires, 1991).

The petroleum spray oils with application rates between 100x and 200x are frequently applied on citrus to control Arrowhead scale, Unaspis yanonensis (Kuwama) in Jeju (RDA, 2008). These have also been found to be effective against citrus red mite, Panonychus citri (Herron et al., 1995) and citrus leaf miner, Phyllocnistis citrella (Beattie et al., 1995a, b). Furthermore, they can also improve integrated pest management systems because petroleum oils do not severely affect populations of beneficial arthropods, although predators and parasites may be killed on contact when sprayed directly (Davidson et al., 1991). Both the direct application as well as the deposits of petroleum spray oil were found to be highly effective in controlling Aphis gossypii (Najar-Rodriguez et al., 2007a, b).

Though, anoxia is considered as the most common physical mode of action of these oils that act by blocking the spiracles, accumulating in the tracheae and thus suffocating the insect (Davidson et al., 1991), petroleum spray oils have been suggested to have effects other than suffocation, including 
desiccation through water loss (Wigglesworth, 1941; Ebeling, 1974), solubilisation of cell membranes (Van Overbeek and Blondeau, 1954) and disruption to the nervous system (Taverner et al., 2001). Direct toxic effects of an $\mathrm{nC} 15$ oil to the peripheral nerves of light brown apple moth, Epiphyas postvittania Walker have been demonstrated by Taverner et al. (2001). Negative behavioural effects on the feeding and oviposition behaviour of Helicoverpa adults (Mensah et al., 1995) and citrus leaf miner, Phyllocnistis citrella Stainton (Beattie et al., 1995a) have also been recognized and quantified.

The commercial formulation of the petroleum spray oil was found to be highly effective in controlling the turnip aphid at concentration as low as $0.75 \%$. It did not show any apparent toxic effect on the honeybees as well as ladybird beetles in the field as well as laboratory. Recently, Najar-Rodriguez et al. (2008) have demonstrated that modern petroleum spray oils show their effect on the nervous system of insects. Since petroleum spray oils show their effect on the nerve activity of insects, thus, they could be integrated into resistance management strategies especially for synthetic insecticides that target central nervous system of insects such as pyrethroids and organophosphates. Though these insecticides also act on central nervous system but they kill the insect in different ways than the oils. The oil could be applied in conjunction with such synthetic insecticides to kill the resistant individuals that survive the insecticide application. No case for resistance to petroleum spray oils has ever been documented in more than a century of use (Davidson et al., 1991), which further confirms the suitability of petroleum spray oils as effective components of insecticide resistance management programs. The petroleum spray oils could also be used as a means of increasing the penetration and toxicity of new botanical pesticides or as a tool to deliver the toxins to their site of action, if this includes the central nervous system (Najar-Rodriguez et al., 2008).

\section{Conclusion}

Petroleum spray oil @ 1.75\% can be used as an alternative to chemical insecticides for the management of aphids on rapeseed-mustard. It offers an environment friendly pest management strategy to which no case of resistance by any pest has been reported so far. Thus, a pest control product based on petroleum spray oil could be promising for the control of soft bodied insects such as turnip/mustard aphid and can be incorporated in Integrated Pest Management (IPM) program.

\section{References}

Agnello, A.M., 2002. Petroleum derived spray oils: chemistry, history, refining and formulation. In: Beattie, G.A.C., Watson, D.M., Stevens, M.L., Rae, D.J., Spooner-Hart, R.N. (Eds.), Spray oils beyond 2000. University of
Western Sydney, Sydney, 2-18.

Anonymous, 1991. Canada 1991 Pesticide Use. Agricultural Information Service Limited, London, UK, 50.

Atri, C., Kumar, B., Kumar, H., Kumar, S., Sharma, S., Banga, S.S., 2012. Development and characterization of Brassica juncea-fruticulosa introgression lines exhibiting resistance to mustard aphid (Lipaphis erysimi Kalt.). BMC Genetics 13, 104. doi: 10.1186/1471-2156-13-104. Beattie, G.A.C., 1990. Citrus petroleum spray oils. Agfact H2. AE.5. NSW Agriculture and Fisheries, 4.

Beattie, G.A.C., 1991. The use of petroleum oil sprays in citrus and other horticultural crops. Proceedings of the First National Conference of the Australian Society of Horticultural Science 1, 351-62.

Beattie, G.A.C., 1989. Citrus leaf miner. NSW Agriculture and Fisheries, Agfact, H2. AE, 41-4.

Beattie, G.A.C., Smith, D., 1990. Integrated pest management: sustainable pest control for the future based on the past? Journal of International Society of Citriculture 96, 51-58.

Beattie, G.A.C., Liu, Z.M., Watson, D.M., Clift, A.D., Jiang, L., 1995a. Evaluation of petroleum spray oils and polysaccharides for control of citrus leaf miner, Phyllocnistis citrella Stainton (Lepidoptera: Gracillariidae). Australian Journal of Entomology 34, 349-53.

Beattie, G.A.C., Somsook, V., Watson, D.M., Clift, A.D., Jiang, L., 1995b. Field evaluation of Steinernema carpocapsae (Weiser) (Rhabditidae: Steinernematidae) and selected chemicals for control of citrus leaf miner, Phyllocnistis citrella Stainton (Lepidoptera: Gracillariidae). Australian Journal of Entomology 34, 335-342.

Davidson, N., Dibble, J., Flint, M., Marer, P., Guye, A., 1991. Managing insects and mites with spray oils. IPM Education and Publications.

Ebeling, W., 1974. Permeability of insect cuticle, $2^{\text {nd }}$ edn. In: Rockstein, M. (Ed.), The Physiology of Insecta, Vol. VI Academic Press, London.

FAOSTAT, 2009. Publically available databases from: Food and Agricultural Organization of the United Nations (FAO). Available at http://faostat.fao.org/.

Furness, G.O., Maelzer, D.A., 1981. The phytotoxicity of narrow distillation range petroleum spraying oils to Valencia orange trees in South Australia: I. The influence of distillation temperature and spray timing on yield and alternate cropping. Pesticide Science 12, 593-602.

Herron, G.A., Beattie, G.A.C., Parkers, R.A., Barchia, I., 1995. Potter spray tower bioassay of selected citrus pests to petroleum spray oil. Australian Journal of Entomology $34,255-263$.

Kumar, S., Atri, C., Sangha, M.K., Banga, S.S., 2011. 
Screening of wild crucifers for resistance to mustard aphid, Lipaphis erysimi (Kaltenbach) and attempt at introgression of resistance gene(s) from Brassica fruticulosa to Brassica juncea. Euphytica 179, 461-470. doi: 10.1007/s10681-011-0351-z.

Lawson, D.S., Weires, R.W., 1991. Management of European red mite (Acari: Tetranichidae) and several aphid species on apple with petroleum oils and an insecticidal soap. Journal of Economic Entomology 84, 1550-1557.

LeCoz, C., Ducombs, G., 2006. Plants and plant products. In: Frosch, P.J., Menne, T., Lepottevin, J.P. (Eds.), Contact Dermatitis. $4^{\text {th }}$ edn, Springer Verlag, Berlin-Heidelberg, Germany, 751-800.

Martin, H., Woodcock, D., 1983. The Scientific Principles of Crop Protection, Edward Arnold, London, 486.

Mensah, R., Harris, W., Beattie, G., 1995. Response of Helicoverpa spp. and its natural enemies to petroleum spray oils in cotton. Entomophaga 40, 263-272.

Miller, R.L., 1983. Spray oil insecticides effectively control some insects and mites. American Nurseryman 158, 37-43.

Najar-Rodriguez, A.J., Lavidis, N.A., Mensah, R.K., Choy, P.T., Walter, G.H., 2008. The toxicological effects of petroleum spray oils on insects-Evidence for an alternative mode of action and possible new control options. Food and Chemical Toxicology 46, 3003-3014.

Najar-Rodriguez, A.J., Walter, G.H., Mensah, R.K., 2007a. The efficacy of a petroleum spray oil against Aphis gossypii Glover on cotton. Part 1: Mortality rates and sources of variation. Pest Management Science 63, 586-595.

Najar-Rodriguez, A.J., Walter, G.H., Mensah, R.K., 2007b. The efficacy of a petroleum spray oil against Aphis gossypii Glover on cotton. Part 2: Indirect effects of oil deposits. Pest Management Science 63, 596-607.

Neubauer, I., 1981. Low volume oil sprays to control the soft scale, Ceroplastes floridensis Comstock, in citrus trees in Israel. Proceedings of International Society of Citruculture 2, 614-615.

Nicetic, O., Watson, D.M., Beattie, G.A.C., Meats, A., Zheng,
J., 2001. Integrated pest management of two-spotted mite Tetranychus urticae on greenhouse roses using petroleum spray oil and the predatory mite Phytoseiulus persimilis. Experimental and Applied Acarology 25, 37-53.

Ohkubo, N., 1981. Role of petroleum oil sprays in an integrated pest management system of citrus crops in Japan. Proceedings of International Society of Citriculture 2, 611-614.

OPSTAT, 2009. Statistical Package for Agricultural Research Workers. Available at www.hau.ernet.in/link/spas.htm

PAU, 2009. Package of practices for crops of Punjab: Rabi 2009-10. Directorate of Extension Education, Punjab Agricultural University, Ludhiana, 45-57.

Priyamedha, Singh, B.K., Thomas, L., Bala, M., Singh, V.V., Singh, D., 2015. Status and perspective of canola quality rapeseed-mustard cultivation in India: a review. Journal of Oilseed Brassica 6(1), 142-151.

RDA (Rural Development Administration), 2008. Development of IPM technology for Environment-friendly Managed Citrus Orchards. Site Joint Cooperating Agricultural Research-promoting project, Publication No.: 111390000-002050-01. 136.

Riehl, L.A., 1981. Fundamental consideration and current development in the production and use of petroleum oils. Proceedings of the Fourth International Society of Citriculture, Tokyo, Japan, 601-607.

Singh, C.P., Sachan, G.C., 1991. Assessment of yield losses in yellow sarson due to mustard aphid, Lipaphis erysimi (Kalt). Journal of Oilseeds Research 11, 179-184.

Taverner, P., Gunning, R., Kolesik, P., Bailey, P., Inceoglu, A.B., Roush, R., 2001. Evidence for direct neural toxicity of a light" oil on the peripheral nerves of lightbrown apple moth. Pesticide Biochemistry and Physiology 69, 153-165.

Van Overbeek, J., Blondeau, R., 1954. Mode of action of phytotoxic oils. Weeds 3, 55-65.

Wigglesworth, V., 1941. Oils aiding loss of water from the cuticle. Nature 147, 116. 